\title{
Recombinant luteinizing hormone supplementation in assisted reproductive technology: a review of literature
}

\author{
Maryam Eftekhar(i) and Nasim Tabibnejad* (i)
}

\begin{abstract}
Background: Luteinizing hormone (LH) has the main role in ovarian function in both natural and artificial cycles. A normal LH concentration during controlled ovarian hyperstimulation is positively correlated to the number and quality of retrieved oocytes and resulting embryos.

Main body of the abstract: In this study, we reviewed whether rLH administration, adjunct to the ovarian stimulation regimen, could improve clinical outcomes. The literature review showed that rLH supplementation improves assisted reproductive technology (ART) outcomes among women with hypogonadotropic hypogonadism, and hyporesponsive women to follicle-stimulating hormone monotherapy. Besides, rLH supplementation has advantages for poor responder women 36-39 years of age. Even though the data suggested no priority regarding the LH source for improving ART outcome, women with different LH polymorphisms who did not respond similarly to ovarian stimulation may benefit from adjuvant rLH therapy.
\end{abstract}

Conclusion: $\mathrm{rLH}$ usage for improving ART outcome should be scrutinized via well-designed studies considering the subgroups of infertile women who benefit the most from rLH adjuvant therapy, the type of ovarian stimulation protocol to which rLH would be added, and also the exact dosage, as well as the proper timing (during or prior to a cycle).

Keywords: Recombinant luteinizing hormone, Assisted reproductive technology, Adjuvant therapy

\section{Background}

Gonadotropin therapy has a key role in ovarian stimulation. Extracting gonadotropins from urine as well as the production of recombinant human gonadotropins, and setting them commercially available was an evolution in infertility treatment. Follicle-stimulating hormone (FSH) mainly regulates follicular growth, while luteinizing hormone (LH) has varied effects on the different phases of both natural and assisted reproductive cycles. LH is a heterodimer glycoprotein produced by the anterior pituitary gland under the influence of gonadotropin-releasing hormone $(\mathrm{GnRH})$, and the key functions of $\mathrm{LH}$ are

*Correspondence: nasimtabibnejad@gmail.com

Research and Clinical Center for Infertility, Yazd Reproductive Sciences Institute, Bouali Ave, Safaeye, Yazd 8916877391, Iran promoting follicular development, steroidogenesis, and growth of the leading follicle [1].

Typically, both GnRH agonists and gonadotropins are used during controlled ovarian stimulation (COS) for preventing premature LH surge and to simplify folliculogenesis. Even though the regimen for ovarian stimulation highly depends on the patient's clinical characteristics [2], the optimal LH level achieved after GnRH administration is still controversial among subgroups of infertile patients [3]. In addition, it is not clear whether all categories of women undergoing COS benefit from exogenous LH supplementation. In this review, we focused on the classifications of infertile women who may need LH supplementation during $\mathrm{COS}$ and also the role of $\mathrm{LH}$ in the treatment protocols. Moreover, the role of different $\mathrm{LH}$ preparation, as well as the LH receptor variation, was reviewed. 


\section{Main text}

We performed a narrative literature search in the Medline/PubMed, Google Scholar, and Scopus databases. The end date for the search was June 2021. Our search strategy was based on the following keywords, alone or combined, "luteinizing hormone", "recombinant LH", "rLH", "rhLH", "ovulation induction", "assisted reproductive technology", "ART", "in vitro fertilization", "IVF", "Intracytoplasmic sperm injection", "ICSI", "anovulation", "poor responders", "hyporesponse", "LH preparations", "LHCG receptor", and "polymorphism".

\section{A brief overview}

$\mathrm{LH}$, a glycoprotein that is synthesized periodically by the pituitary gland, coupled with FSH, is considered the main reproductive hormone. $\mathrm{LH}$ direct binding to its receptor on the surface of the target cells on the ovaries induces a stream of molecular events [4]. This procedure continues with the activation of specific signaling pathways and leads to gene expression. Both LH and human chorionic gonadotropin (hCG) have long been thought to be equivalent because of their common receptor, which is mostly expressed in the gonads and stimulates the classical cAMP/protein kinase A steroidogenic pathway [5].

LH stimulates the theca cells, which are located on the outer layer of the primary follicles, to secrete androgens which are in turn converted to estrogens during folliculogenesis [6]. The mid-cycle LH surge motivates final oocyte maturation followed by ovulation. The oocyte meiosis resumption occurs by the completion of meiosis I and initiating meiosis II along with the separation of the oocyte-cumulus complex (COC) from the follicle wall leading to ovulation [7]. Both experimental and clinical documents represent that there is a "threshold" for LH levels to promote normal follicular development and oocyte maturation [8,9]. It is stated that the expected amount of $\mathrm{LH}$ required for optimum efficacy is very low, and usually less than $1 \%$ of $\mathrm{LH}$ receptors on the ovarian follicles need to be occupied for achieving the highest steroidogenic response [10]. This perception was originated from old pharmacological in vitro studies on the effect of hCG on rodents' Leydig cells. However, new evidence criticizes this concept by indicating differences in rodent gonadotropin-responsive cells as opposed to human ones [11].

On the other hand, overexposure to LH has a detrimental effect on pre-ovulatory follicular development. Depending on the follicular developing stage, excessive LH concentrations result in premature luteinized follicles and follicular atresia $[10,12,13]$. It is stated that a limited amount of LH is necessary for normal follicular growth. In other words, a "ceiling" can be defined for LH level, which is higher for mature follicles than immature ones and LH concentrations above the "ceiling" have adverse effects on the growing follicles [8]. As a result, the LH window during the follicular phase of the menstrual cycle is considered LH levels between "threshold" and "ceiling" values [8].

\section{LH in controlled ovarian hyperstimulation cycles}

$\mathrm{COS}$ is considered one of the main factors to achieve a desirable outcome in assisted reproductive technology (ART) cycles. Moreover, FSH and LH play a synergistic role in folliculogenesis during stimulated cycles. At first, $\mathrm{GnRH}$ analogs and gonadotropins were used to reduce unexpected LH surges during COS. Throughout the progress of ovarian stimulation in in vitro fertilization (IVF) cycles, the LH potential value and its effective dose along with the efficacy of different LH forms have been assessed by several studies [14-17]. hCG is the drug of choice for inducing LH activity in the clinical use, especially in ART cycles, and lately recombinant LH (rLH) has become available for clinical practice [5].

Recently, a large observational study among poor prognosis women who used the combination of $\mathrm{LH}$ and FSH during COS showed that adding LH to FSH significantly increased the mean of retrieved oocytes and cumulative embryos [16]. Exogenous LH may have advantages for women with long $\mathrm{GnRH}$ agonist downregulation or those who were treated with GnRH antagonist protocol as well as women of advanced reproductive age and patients with hypogonadotropic hypogonadism.

\section{rLH in hypothalamic hypogonadism women; WHO type I anovulation}

One incontrovertible group of patients who may benefit from LH supplementation is hypogonadotropic hypogonadism $(\mathrm{HH})$ women or World Health Organization (WHO) type I anovulation with a prevalence of $10 \%$ of women at reproductive age [18]. These patients have no endogenous FSH or LH due to the absence of hypothalamic-pituitary activity and FSH administration alone in these women does not result in adequate follicular growth and good-quality oocytes [19]. FSH promotes several follicle developments, but ovarian endocrine irregularities and low oocyte fertilization rates were observed due to the lack of LH activity [20]. The abovementioned ideas on gonadotrophic control of folliculogenesis indicate that despite normal follicular development stimulated by FSH, the exact amount of exogenous $\mathrm{LH}$ is required for optimizing ovarian stimulation. Thus, co-administration of FSH and LH in an ideal ratio could be beneficial for attaining optimal results. $\mathrm{rLH}$ therefore seems to be an ideal adjuvant therapy besides $\mathrm{rFSH}$ in women with $\mathrm{HH}$. Several studies confirmed the positive effect of $\mathrm{rLH}$ in addition to recombinant $\mathrm{FSH}(\mathrm{rFSH})$ on follicular 
growth, maturation, and pregnancy rate among women affected by $\mathrm{HH}$ [21-24]. A daily dose of $75 \mathrm{IU}$ r-LH was effective in the majority of patients, although the dose of $225 \mathrm{IU}$ may be required in some cases [25]. Overall, it is indicated that the proper dose for achieving follicular development, optimum serum estradiol level, and optimal endometrial thickness is $150 \mathrm{IU}$ rFSH with $75 \mathrm{IU} \mathrm{rLH}$ daily by a 2:1 ratio [26]. Adding rLH to rFSH during COS is also identified as a cost-effective method for pregnancy, which also improves the quality of life in $\mathrm{HH}$ women [27, 28]. On the other hand, in many cases, pretreatment with rLH prior to beginning FSH was valuable to increase the number of antral follicles and pregnancy rates [29]. The mechanism may be defined by the intra-ovarian follicular androgens that stimulate the aromatase activity of antral follicles $[9,30]$. Consequently, it is assumed that increasing LH-induced androgen prior to ovarian stimulation results in an elevation of follicular recruitment and pregnancy rates $[31,32]$. Therefore, it could be concluded that $\mathrm{rLH}$ administration in proper dose and appropriate FSH:LH ratio is valuable for patients diagnosed with $\mathrm{HH}$.

\section{rLH in WHO type II anovulation}

The majority of infertile anovulatory women are categorized as normogonadotropic anovulatory or WHO group II anovulation, and most of these patients are diagnosed with polycystic ovary syndrome (PCOS) [33]. Hormonal imbalance, elevating LH levels, and abnormal intraovarian regulation of FSH are typical aspects of PCOS patients. Both human menopausal gonadotropin (hMG) and FSH have been administered effectively for ovulation induction in these patients [34]. Generally, it is assumed that in the presence of elevated endogenous LH, FSH alone is conceptually better in PCOS women [30, 35-37]. With this regard, the possible effect of $\mathrm{LH}$ administration on follicular development in this type of patients should be investigated clinically. The data on the effect of additional LH on ART outcome is limited among women with WHO type II anovulation. However, a randomized study showed that rLH administration in WHO type II anovulatory patients who over-responded to FSH during COS increases the percentage of women with developing single dominant follicle $\geq 16 \mathrm{~mm}$. This study also confirmed the "ceiling" theory that stated the administration of LH in a high dose could suppress follicular growth [38]. The "LH ceiling" concept was also supported by another study in which administration of rLH alone triggered arrest of follicular growth among the majority of patients in the WHO type II anovulatory group [39]. In addition, another study showed different LH levels among PCOS subgroups and concluded that adjusting LH administration to minimize the negative effect of $\mathrm{LH}$ in hyperandrogenic PCOS women should be considered [40].
It seems that low FSH supply plus the administration of rLH under the "ceiling" during ovarian stimulation regimens results in reasonable E2 levels and follicular development in women with WHO type II anovulatory. However, further evaluations are required to determine the strategies for LH administration in PCOS subgroups.

\section{rLH in GnRH agonist cycles}

GnRH agonists are administered besides gonadotropins for preventing $\mathrm{LH}$ premature surge during COS. Without using $\mathrm{GnRH}$ analogs, an LH rise may occur prematurely in approximately $20 \%$ of stimulated IVF cycles [41]. It has been evidenced that throughout ovarian stimulation with both FSH and GnRH agonists, endogenous LH concentration is reduced to the lowest level during the late stimulation phase [42]. Therefore, it could be expected that adding LH to the ovarian stimulation protocol improves cycle outcome. The results of meta-analyses that assess the value of $\mathrm{rLH}$ supplementation for $\mathrm{COS}$ in $\mathrm{rFSH}$ and $\mathrm{GnRH}$ agonist protocol among the general population of infertile women showed fewer days of stimulation, a lower dosage of r-FSH administered and a higher serum estradiol level on the day of hCG administration. Nonetheless, there were no differences in the number of retrieved oocytes, mature oocytes, clinical pregnancy rate, implantation rate, miscarriage, and live birth rates [43-46]. Conversely, another meta-analysis presented a better pregnancy rate by $\mathrm{FSH}+\mathrm{LH}$ compared with $\mathrm{FSH}$ alone in GnRH agonist protocols [47]. Besides, two other meta-analyses showed that LH plus rFSH in GnRH agonist cycles may improve implantation and clinical pregnancy in women $\geq 35$ years [48] and poor responder patients [49]. In summary, it seems that rLH supplementation may have beneficial effects on pregnancy outcome in GnRH agonist cycles. However, the effect of rLH administration in following subgroups of infertile women undergoing $\mathrm{GnRH}$ agonist protocol should be considered independently.

\section{rLH in GnRH antagonist cycles}

$\mathrm{GnRH}$ antagonists were introduced into ART cycles with high effectiveness and lower side effects than GnRH agonists for preventing premature LH surges. A shorter duration of administration, lack of vasomotor symptoms, prevention of ovarian hyperstimulation, and a remarkable lower dose of gonadotropin per cycle, make GnRH antagonists not only well-tolerated by patients but also clinicians' preference [41]. Adding $\mathrm{LH}$ to the $\mathrm{GnRH}$ protocol for improving ART outcomes has been investigated in several studies. One study among egg donors reported a significantly higher percentage of metaphase II (MII) oocytes, 2 pronuclears and embryo quality in the group of donors who were undergoing $\mathrm{GnRH}$ antagonist 
supplemented with r-LH. Moreover, the implantation rate was higher in recipients who received embryos from donors treated with rLH [50]. Furthermore, 253 women of $>35$ years who were undergoing IVF or intracytoplasmic sperm injection (ICSI) and received $\mathrm{rFSH}$ plus a $\mathrm{GnRH}$ antagonist for ovulation induction, were randomly supplemented with exogenous $\mathrm{LH}$ or $\mathrm{rFSH}$ alone. The result showed that adding $\mathrm{LH}$ during the second part of the follicular phase could not improve pregnancy rates, implantation rate, or ovarian response in these women [51]. In a similar study, Bosch et al. assessed the effect of administration of rFSH alone versus $\mathrm{rFSH}+\mathrm{rLH}$ on IVF outcome. Two groups of women undergoing IVF were divided into two subgroups: < 35 years $(n=380)$ and $36-39$ years $(n=340)$. As a result, LH supplementation significantly improved the implantation rate, but not the ongoing pregnancy rate among patients aged 36 to 39 years [52]. It may be due to the higher susceptibility of women older than 35 to LH suppression in GnRH antagonist cycles, and consequently, these women are more likely to benefit from exogenous LH [53]. Another study evaluated 127 infertile patients undergoing IVF/ICSI who were randomized to receive $\mathrm{rFSH}$ or $\mathrm{rFSH}$ plus $\mathrm{rLH}$ for ovarian stimulation during a $\mathrm{GnRH}$-antagonist cycle. The authors found that exogenous LH did not shorten the time for ovulation induction. Serum E2 and LH levels were significantly higher in the rLH-supplemented group, but the other treatment parameters did not differ significantly between the two study groups [14]. LeviSetti et al. randomized 40 infertile normo-ovulatory women undergoing GnRH antagonist for ICSI into two groups: 20 women received $\mathrm{rFSH}$ alone, and 20 women were treated with $\mathrm{rFSH}$ plus $\mathrm{rLH}$. The results indicated that the use of rLH in addition to rFSH may prevent E2 drop after $\mathrm{GnRH}$ antagonist administration, but did not improve oocyte number, oocyte maturation, embryo quality as well as fertilization rate, pregnancy, or implantation rates [54]. A meta-analysis aimed to evaluate the effect of additional LH in women undergoing IVF/ICSI who were stimulated with $\mathrm{GnRH}$ antagonist protocol. This review assessed four of the aforementioned studies. The result showed no significant differences between the $\mathrm{rLH}$ supplementation group and the $\mathrm{rFSH}$ alone group regarding clinical and ongoing pregnancy [42]. Furthermore, another meta-analysis stated that LH supplementation during an antagonist protocol could not increase the number of mature retrieved oocytes, or ART outcomes [53]. Therefore, it seems that rLH supplementation may not be appropriate for improving outcome in GnRH antagonist cycles. Nonetheless, rLH co-treatment may be valuable for a specific population of infertile women undergoing $\mathrm{GnRH}$ protocol, such as women aged $36-39$ years.

\section{rLH in women of advanced reproductive age}

It is well known that the IVF success rate declines with aging and low ovarian sensitivity to gonadotropins. The problem is managed by the clinicians through increasing gonadotropin dose or adding LH supplementation to the treatment protocol [33]. Earlier studies indicated that LH adjuvant therapy in downregulated women of advanced reproductive age may improve ART outcomes $[55,56]$. There are studies with conflicting results that evaluated the effect of $\mathrm{rLH}$ supplementation in GnRH antagonist protocol among women $\geq 35$ years $[51,52,57,58]$. Some studies found that LH supplementation could not increase the implantation, pregnancy or live birth rates in women of 35 years and older who have undergone $\mathrm{GnRH}$ antagonist in IVF/ICSI cycles [51, 57, 58]. They proposed that endogenous serum LH concentrations after GnRH antagonist administration are adequate for inducing late follicular ovarian steroidogenesis in women $\geq 35$ years [58]. However, Bosch et al. claimed that implantation rate was significantly increased by using $\mathrm{rLH}$ in women aged between 36 and 39 years [52]. Besides, it is stated that adding $\mathrm{rLH}$ to $\mathrm{rFSH}$ under pituitary downregulation would improve the number of oocytes but did not affect the number of embryos or pregnancy rate [59]. A systematic review and meta-analysis on women of advanced reproductive age concluded that adding $\mathrm{rLH}$ to the treatment protocol during ART cycles could improve implantation and clinical pregnancy rates with no effect on the number of the retrieved oocytes [48]. More recently, a meta-analysis showed a higher oocyte yield in $\mathrm{rFSH}$ monotherapy versus an improvement in implantation rate and clinical pregnancy rate among women between 35 and 40 years of age undergoing $\mathrm{rLH} / \mathrm{rFSH}$ co-treatment during COS [60].

The increasing implantation and clinical pregnancy may be due to the increased oocyte competence or developed endometrial receptivity. Recombinant LH supplementation prevents cumulous cell apoptosis better than FSH-only administration, which improved oocyte quality in LH-supplemented cycles [61]. The summary of abovementioned studies indicated that rLH administration has a beneficial effect on both implantation and clinical pregnancy rates in women aged 36-39 years, regardless of the ovarian stimulation protocol. Nevertheless, the beneficial effect of rLH on improving ART outcome was not reported in women aged over 40 years [2].

\section{rLH in hyporesponse women to FSH monotherapy}

Hyporesponsive patients are normogonadotropic women with an apparent normal ovarian reserve and a low response to $\mathrm{rFSH}$ during COS. This category shows a steady response with regard to follicular growth and E2 level during ovarian stimulation [2]. These women, who 
constitute $10-14 \%$ of patients undergoing GnRH agonist protocol, need high doses of rFSH to attain an adequate number of oocytes $[48,62]$. Randomized clinical trials (RCTs) showed a significantly higher number of oocytes as well as implantation and pregnancy rates among hyporesponsive women who received $\mathrm{rLH}$ supplementation in addition to $\mathrm{rFSH}$ rather than those treated with $\mathrm{rFSH}$ alone or women with increasing the dose of $\mathrm{rFSH}$ [61-63]. A systematic review and meta-analysis confirmed these results among hyporesponsive women treated with $\mathrm{rLH}$ compared with hyporesponders who underwent FSH monotherapy [64].

Even though the mechanism behind the low response in these patients is not entirely identified, it may be due to the excessive suppression of endogenous $\mathrm{LH}$ after downregulation with $\mathrm{GnRH}$ agonists. It is also claimed that the low response in these patients may be related to a mutation in both LH and FSH receptors [65] as women with FSH receptor Ser/680 variants require greater doses of exogenous FSH for optimal ovarian response $[2,66]$. Lastly, according to the existing evidence, rLH administration during $\mathrm{GnRH}$ agonist pituitary downregulation has more beneficial effects on ART outcome rather than increasing FSH dose in women who show a hyporesponse to FSH monotherapy. In other words, rLH supplementation from day 7 of the stimulation cycle could rescue the cycle in patients who did not respond appropriately at the beginning of the cycle.

\section{$\mathrm{rLH}$ in poor responders}

Poor ovarian response to controlled ovarian hyperstimulation is still one of the most challenging subjects in assisted reproduction. Almost $9-24 \%$ of women undergoing IVF are categorized as poor responders [67]. Some studies suggested the necessity of additional LH in poor responders during ovarian stimulation with short and long protocols of $\mathrm{GnRH}$ agonists [68, 69]. LH supplementation seems to be advantageous for poor responders with a positive effect on follicular and endometrial conditions, which improves cycle outcome [70]. The effect of adding rLH to the ovarian stimulation regimen was investigated in different subgroups of poor responders. The results showed no significant differences in terms of the implantation, fertilization, and pregnancy rates along with the number of retrieved and MII oocytes, when $\mathrm{rLH}$ was added to COS protocols [58, 68, 71, 72]. Only one study with a limited sample size claimed that rLH administration before starting ovarian stimulation could increase the live birth rate [73]. In addition, a large study including 4828 ICSI cycles of poor responders categorized according to the Patient-Oriented Strategies Encompassing IndividualizeD Oocyte Number (POSEIDON) [74], suggested LH supplementation for improving
ICSI outcome in women with an expected low response to ovarian stimulation [16]. Indeed, the combination of $\mathrm{LH}$ and FSH is useful in the suboptimal responders (4-9 oocytes) with poor baseline characteristics such as higher age and basal FSH levels as well as lower anti-Mullerian hormone and antral follicle count [16]. Summarizing the data of RCTs showed that hypo-responder women, for instance, groups 1 and 2 POSEIDON who have 1-9 oocytes, may benefit from rLH during COS [75].

In general, the evidence available on the clinical outcome after $\mathrm{r}-\mathrm{hLH}$ supplementation in poor responder women is indecisive, mostly because of the high heterogeneity in the definition of poor response among studies [2].

In summary, the confirmation of positive clinical effect of adding rLH to the ovarian stimulation protocol in poor responders needs more investigation considering homogenous subgroups and gonadotropin receptors.

\section{rLH in women at risk for ovarian hyperstimulation syndrome}

Ovarian hyperstimulation syndrome (OHSS) is a lifethreatening iatrogenic complication, with a prevalence of 0.5 to $1 \%$ per ART cycle [76]. The mid-cycle rLH administration can be efficient for preventing OHSS. It is evidenced that the growth of several small follicles during ovulation induction increases the possibility of OHSS [77]. Therefore, the LH administration which reduces the number of $<10$ - $\mathrm{mm}$ follicles could decrease the risk of OHSS [78]. In an RCT, Caserta et al. compared 999 infertile women $\leq 40$ years undergoing long GnRH agonist protocol with regard to OHSS development. In this study, women treated with rLH plus rFSH were compared with those with the administration of rFSH alone. The result showed that women supplemented with rLH had a significantly higher clinical pregnancy rate. Moreover, adding rLH caused lower developing clinical OHSS and a chance for cycle cancellation due to the risk of OHSS as well [79].

\section{Different sources of LH preparation}

Two common commercially available sources of LH are hMG (a urinary-derived product which holds both FSH and LH in equal concentrations of $75 \mathrm{IU}$ ) and rLH (lutropin alfa). More recently, a highly purified form of hMG (HP-hMG) has been offered, with 10 IU more hCG and 5 IU less LH than the other hMG preparations. Later, rFSH and rLH were pooled in a single preparation (Follitropin alfa/lutropin alfa 150 IU/75 IU), in which two gonadotropins could be simply injected at a 2:1 ratio [80].

Both rLH and HP-hMG bind the LH receptor. However, it seems that the mechanisms of receptor activation are different due to the longer hCG half-life and its higher binding affinity to the $\mathrm{LH}$ receptors [81]. It is also 
shown that adding rLH and hMG preparations to ovarian stimulation regimens induce upregulation and downregulation in several cumulus gene clusters leading to the activation of different pathways $[82,83]$. The main question is whether the addition of various LH forms during COS affects ART outcome differently. Many studies examined the effect of different sources of LH including the abovementioned types along with recombinant hCG (rhCG) and urinary hCG (uhCG) during ART cycles. A double-blinded randomized trial compared the effect of adding rLH to uhCG supplementation in poor responders undergoing GnRH antagonist protocol in IVF cycles. The result showed similar cancellation rates, numbers of retrieved oocytes, fertilization rates, the numbers of embryos obtained as well as implantation, clinical pregnancy, and live birth rates [84]. Moreover, some studies that compared the effect of adding hMG to rLH supplementation in stimulation regimen, reported no significant differences regarding the total and daily dose of gonadotropin, or clinical pregnancy and live birth rates; however, the number of retrieved oocytes was significantly higher in the application of rLH in comparison with using hMG $[85,86]$. Recently, a meta-analysis reviewed 70 studies and concluded that the number of MII oocytes did not vary when FSH alone was compared to FSH plus LH, FSH plus hCG, or hMG. The number of embryos obtained and implantation rate were higher when hMG was administered as a substitute for FSH alone. The pregnancy rate was significantly higher in patients who received FSH plus LH in comparison with the other groups. The live birth rate did not differ significantly between groups [47]. Furthermore, a recent review assessed the effect of different sources of LH during COS protocols on cycle characteristics and its outcome in 11 studies. The authors concluded that no statistically significant differences were detected in ovarian stimulation variables and clinical pregnancy and live birth rates when hMG was compared with recombinant FSH plus recombinant LH [80]. Overall, considering all the RCTs, observational and retrospective studies, stronger evidence is necessary for approving the superiority of one source of LH to the others.

\section{LH receptor and ART outcome}

Both FSH and LH induce the gonadotropic effects through their specific receptors. The FSH receptor (FSHR) is located on ovarian granulosa cells, while the luteinizing hormone/human chorionic gonadotropin receptor (LHCGR) is found on granulosa, theca, and luteal cells [87]. It is stated that LH receptor expression has a crucial role in follicular development, ovulation, and the corpus luteum function [88]. The data on LHCGR expression and its correlation with ART outcomes are limited. It has been shown that women undergoing IVF who are carriers of V-betaLH, a common genetic polymorphism of $\mathrm{LH}$, demonstrate suboptimal ovarian response to long GnRH-agonist protocol [89]. One study introduced $L H C G R$ gene expression profiling as a predictor for ovarian response, duration of stimulation and also pregnancy achievement. LHCGR gene expression was assessed among 40 women who received a 5-day pretreatment with rLH before starting long luteal GnRH-agonist protocol for ICSI. A statistically significant negative association was found between LHCGR gene expression and the duration of ovarian stimulation. Moreover, six of the seven women who became pregnant expressed at least two specific $L H C G R$ variants (735 bp, $621 \mathrm{bp)}$ [90]. In another study, Yin et al. showed an independent correlation between the slow ovarian response with the LHCGR genotypes of rs13405728, the initial gonadotropin dose, and the required dose of luteinizing hormone [91]. Furthermore, the LHCGR expression was reported to be associated with the follicle size and follicular development such that its expression was significantly higher in MII oocytes than in metaphase I or germinal vesicle oocytes through in vitro maturation process. Besides, overexpression of LHCGR in MII oocytes may be an indicator of oocytes malfunction and following low fertilization capacity [92]. In a large study, women with FSHR (rs6166) and LHCGR (rs2293275) allele G were found to have a 4-fold increased chance of pregnancy versus A carriers of both polymorphisms [93]. On the other hand, some studies reported that LHCGR variants could predict the chance to deliver a live-born baby during IVF. In a cross-sectional study, 348 women undergoing IVF were genotyped in terms of $L H C G R$ variant N312S. The genetic variant compared between the women who conceived after IVF with those who did not. Women who were homozygous for serine showed higher pregnancy rates in comparison with those with homozygous asparagine [94]. In a different study, Lu et al. concluded that IVF using frozen embryo transfer may be a valuable infertility treatment for women with $L H C G R$ mutations. They reported successful pregnancy and live birth in two of the three women affected by pathogenic $L H C G R$ variants (p.Ile585Leufs*16, p.Arg283*, and p.Asn377Asp). All three women showed low estradiol and progesterone levels, enlarged cystic ovaries, oligomenorrhea, and infertility. All women had reasonable oocytes and highquality embryos and following frozen embryo transfer, one woman achieved a successful twin live birth, and the other one gave birth to a healthy baby boy [95]. In summary, only one meta-analysis showed that FSHR (rs6165) and FSHR (rs6166) polymorphisms affect the response to COS and ART outcomes [96]. However, further large studies are needed to confirm the involvement of $L H C G R$ 
expression in follicular and oocyte growth and its predictability for ART success.

\section{Conclusions}

Based on the collected research, rLH supplementation is highly recommended for both groups of patients with hypogonadotropic hypogonadism and also for hyporesponsive women to FSH monotherapy during GnRH agonist cycles. Furthermore, according to the available evidence, co-treatment with rLH has a positive impact on implantation and clinical pregnancy rates during both the $\mathrm{GnRH}$ agonist and antagonist regimen among women of advanced reproductive age between 36 and 39 years. Moreover, rLH support is suggested for women at risk of OHSS as it could decrease the cancelation rate caused by the development of OHSS in these patients. Nonetheless, on the topic of poor responders, rLH administration during COS cycles is still controversial because of the heterogeneity in the poor responder population and also various study designs considering different definitions for poor responder women. On the other hand, the review of the studies has revealed that there is no preference in terms of the LH source for improving ART outcome. Although the available data suggest that women with different LH polymorphisms respond differently to COS, the data is not enough to conclude that women with specific LH polymorphisms may benefit from adjuvant rLH therapy. Nowadays, with the encouraging attitudes toward individualized medicine, the ART regimen would also be directed by patients' characteristics and genetic features. To that end, by continuing to pool the findings of clinical research, a comprehensive and fixed agreement on the proper dose and frequency of LH administration during ovarian stimulation may be achieved. Furthermore, the factors that determine women who would benefit the most from exogenous LH need to be clarified, and also the cost-benefit ratio in the use of rLH among different subgroups of the infertile women should be provided. This will deliver new paths on how in the future different categories of infertile women may benefit from rLH administration during $\mathrm{COS}$ cycles that enhance ovarian response and oocyte and embryo quality in addition to pregnancy outcome.

\footnotetext{
Abbreviations

ART: Assisted reproductive technology; COC: Oocyte-cumulus complex; COS: Controlled ovarian stimulation; FSH: Follicle-stimulating hormone; FSHR: Follicle-stimulating hormone receptor; $\mathrm{GnRH}$ : Gonadotropin-releasing hormone; hCG: Human chorionic gonadotropin; HH: Hypogonadotropic hypogonadism; hMG: Human menopausal gonadotropin; HP-hMG: Highly purified form of hMG; ICSI: Intracytoplasmic sperm injection; IVF: In vitro fertilization; LH: Luteinizing hormone; LHCGR: Luteinizing hormone/human chorionic gonadotropin receptor; MII: Metaphase II; OHSS: Ovarian hyperstimulation syndrome; PCOS: Polycystic ovary syndrome; POSEIDON: PatientOriented Strategies Encompassing IndividualizeD Oocyte Number; RCT:
}

Randomized clinical trials; rFSH: Recombinant follicle-stimulating hormone; rhCG: Recombinant human chorionic gonadotropin; rLH: Recombinant luteinizing hormone; uhCG: Urinary human chorionic gonadotropin; WHO: World Health Organization.

\section{Acknowledgements}

Not applicable

\section{Authors' contributions}

ME and NT made substantial contributions to the conception/design of the work. ME and NT did the database search. ME supervised the research. NT drafted the manuscript. ME and NT critically revised the manuscript. ME and NT read and approved the final version of the manuscript.

\section{Funding}

There was no source of financial support.

Availability of data and materials

Not applicable

\section{Declarations}

Ethics approval and consent to participate

Not applicable

Consent for publication

Not applicable

Competing interests

There is no competing interest to declare.

Received: 23 August 2021 Accepted: 18 September 2021

Published online: 08 October 2021

\section{References}

1. Fritz MASL (2011) Clinical gynecologic endocrinology and infertility, 8th edn. Wolters Kluwer Health/Lippincott Williams \& Wilkins, Philadelphia

2. Alviggi C, Conforti A, Esteves SC, Andersen CY, Bosch E, Bühler K et al (2018) Recombinant luteinizing hormone supplementation in assisted reproductive technology: a systematic review. Fertil Steril 109(4):644-664. https://doi.org/10.1016/j.fertnstert.2018.01.003

3. Esteves SC, Alviggi C (2015) The role of LH in controlled ovarian stimulation. In: Ghumman S, editor. Principles and Practice of Controlled Ovarian Stimulation in Art. New Delhi: Springer India. p. 171-96.

4. Aflatoonian A, Yousefnejad F, Eftekhar M, Mohammadian F (2012) Efficacy of low-dose hCG in late follicular phase in controlled ovarian stimulation using GnRH agonist protocol. Arch Gynecol Obstet 286(3):771-775

5. Casarini L, Santi D, Brigante G, Simoni M (2018) Two hormones for one receptor: evolution, biochemistry, actions, and pathophysiology of LH and hCG. Endocr Rev 39(5):549-592. https://doi.org/10.1210/er. 2018-00065

6. Huhtaniemi I (2018) Encyclopedia of endocrine diseases. Academic Press

7. Eftekhar M, Khalili MA, Rahmani E (2012) The efficacy of recombinant versus urinary HCG in ART outcome. Iran J Reprod Med 10(6):543

8. Balasch J, Fabregues F (2002) Is luteinizing hormone needed for optimal ovulation induction? Curr Opin Obstet Gynecol 14(3):265-274. https:// doi.org/10.1097/00001703-200206000-00004

9. Hillier SG (2001) Gonadotropic control of ovarian follicular growth and development. Mol Cell Endocrinol 179(1-2):39-46. https://doi.org/10. 1016/s0303-7207(01)00469-5

10. Chappel SC, Howles C (1991) Reevaluation of the roles of luteinizing hormone and follicle-stimulating hormone in the ovulatory process. Hum Reprod 6(9):1206-1212. https://doi.org/10.1093/oxfordjournals.humrep. a137513

11. Casarini L, Santi D, Simoni M, Potì F (2018) 'Spare' luteinizing hormone receptors: facts and fiction. Trends Endocrinol Metab 29(4):208-217. https://doi.org/10.1016/j.tem.2018.01.007 
12. Hillier SG (2000) The Parkes lecture: controlled ovarian stimulation in women. J Reprod Fertil 120(2):201-210. https://doi.org/10.1530/jrf.0. 1200201

13. Huirne JA, van Loenen AC, Schats R, McDonnell J, Hompes PG, Schoemaker J et al (2005) Dose-finding study of daily GnRH antagonist for the prevention of premature LH surges in IVF/ICSI patients: optimal changes in LH and progesterone for clinical pregnancy. Hum Reprod 20(2):359367. https://doi.org/10.1093/humrep/deh601

14. Griesinger G, Schultze-Mosgau A, Dafopoulos K, Schroeder A, Schroer A von Otte $S$ et al (2005) Recombinant luteinizing hormone supplementation to recombinant follicle-stimulating hormone induced ovarian hyperstimulation in the $\mathrm{GnRH}$-antagonist multiple-dose protocol. Hum Reprod 20(5):1200-1206. https://doi.org/10.1093/humrep/deh741

15. Humaidan P, Bungum M, Bungum L, Yding AC (2004) Effects of recombinant LH supplementation in women undergoing assisted reproduction with $\mathrm{GnRH}$ agonist down-regulation and stimulation with recombinant FSH: an opening study. Reprod BioMed Online 8(6):635-643. https://doi. org/10.1016/s1472-6483(10)61643-4

16. Levi-Setti PE, Zerbetto I, Baggiani A, Zannoni E, Sacchi L, Smeraldi A et al (2019) An observational retrospective cohort trial on 4,828 IVF cycles evaluating different low prognosis patients following the POSEIDON criteria. Front Endocrinol (Lausanne). 10:282. https://doi.org/10.3389/fendo. 2019.00282

17. Orvieto R (2015) Triggering final follicular maturation--hCG, GnRH-agonist or both, when and to whom? J Ovarian Res 8:60. https://doi.org/10.1186/ s13048-015-0187-6

18. Huseyin K, Berk B, Tolga K, Eser O, Ali G, Murat A (2019) Management of ovulation induction and intrauterine insemination in infertile patients with hypogonadotropic hypogonadism. J Gynecol Obstet Hum Reprod 48(10):833-838. https://doi.org/10.1016/j.jogoh.2019.03.027

19. Ludwig M (2003) Does the addition of luteinizing hormone in ovarian stimulation protocols improve the outcome? Treat Endocrinol 2(5):305-313. https://doi.org/10.2165/00024677-200302050-00002

20. Balasch J, Vidal E, Peñarrubia J, Casamitjana R, Carmona F, Creus M et al (2001) Suppression of LH during ovarian stimulation: analysing threshold values and effects on ovarian response and the outcome of assisted reproduction in down-regulated women stimulated with recombinant FSH. Hum Reprod 16(8):1636-1643. https://doi.org/10.1093/humrep/16.8. 1636

21. Awwad JT, Farra C, Mitri F, Abdallah MA, Jaoudeh MA, Ghazeeri G (2013) Split daily recombinant human LH dose in hypogonadotrophic hypogonadism: a nonrandomized controlled pilot study. Reprod BioMed Online 26(1):88-92. https://doi.org/10.1016/j.rbmo.2012.09.016

22. Carone D, Caropreso C, Vitti A, Chiappetta R (2012) Efficacy of different gonadotropin combinations to support ovulation induction in $\mathrm{WHO}$ type I anovulation infertility: clinical evidences of human recombinant FSH/human recombinant LH in a 2:1 ratio and highly purified human menopausal gonadotropin stimulation protocols. J Endocrinol Investig 35(11):996-1002. https://doi.org/10.3275/8657

23. Kaufmann R, Dunn R, Vaughn T, Hughes G, O'Brien F, Hemsey G et al (2007) Recombinant human luteinizing hormone, lutropin alfa, for the induction of follicular development and pregnancy in profoundly gonadotrophin-deficient women. Clin Endocrinol 67(4):563-569. https:// doi.org/10.1111/j.1365-2265.2007.02925.x

24. Shoham Z, Smith H, Yeko T, O'Brien F, Hemsey G, O'Dea L (2008) Recombinant LH (lutropin alfa) for the treatment of hypogonadotrophic women with profound LH deficiency: a randomized, double-blind, placebocontrolled, proof-of-efficacy study. Clin Endocrinol 69(3):471-478. https:// doi.org/10.1111/j.1365-2265.2008.03299.x

25. Munoz E, Bosch E, Fernandez I, Portela S, Ortiz G, Remohi J et al (2012) The role of LH in ovarian stimulation. Curr Pharm Biotechnol 13(3):409416. https://doi.org/10.2174/138920112799361945

26. Krause BT, Ohlinger R, Haase A (2009) Lutropin alpha, recombinant human luteinizing hormone, for the stimulation of follicular development in profoundly LH-deficient hypogonadotropic hypogonadal women: a review. Biologics 3:337-347. https://doi.org/10.2147/btt.2009.3306

27. Mennini FS, Marcellusi A, Viti R, Bini C, Carosso A, Revelli A et al (2018) Probabilistic cost-effectiveness analysis of controlled ovarian stimulation with recombinant FSH plus recombinant LH vs. human menopausal gonadotropin for women undergoing IVF. Reprod Biol Endocrinol 16(1):68. https://doi.org/10.1186/s12958-018-0386-2
28. Papaleo E, Alviggi C, Colombo GL, Pisanelli C, Ripellino C, Longobardi S et al (2014) Cost-effectiveness analysis on the use of rFSH + rLH for the treatment of anovulation in hypogonadotropic hypogonadal women. Ther Clin Risk Manag 10:479-484. https://doi.org/10.2147/tcrm.s62351

29. Balasch J, Fábregues F, Carmona F, Casamitjana R, Tena-Sempere M (2009) Ovarian luteinizing hormone priming preceding follicle-stimulating hormone stimulation: clinical and endocrine effects in women with long-term hypogonadotropic hypogonadism. J Clin Endocrinol Metab 94(7):2367-2373. https://doi.org/10.1210/jc.2009-0262

30. Hillier SG (1994) Current concepts of the roles of follicle stimulating hormone and luteinizing hormone in folliculogenesis. Hum Reprod 9(2):188-191. https://doi.org/10.1093/oxfordjournals.humrep.a138480

31. Durnerin Cl, Erb K, Fleming R, Hillier H, Hillier SG, Howles CM et al (2008) Effects of recombinant $\mathrm{LH}$ treatment on folliculogenesis and responsiveness to FSH stimulation. Hum Reprod 23(2):421-426. https://doi.org/10. 1093/humrep/dem388

32. Lossl K, Andersen AN, Loft A, Freiesleben NL, Bangsbøll S, Andersen CY (2006) Androgen priming using aromatase inhibitor and hCG during early-follicular-phase GnRH antagonist down-regulation in modified antagonist protocols. Hum Reprod 21(10):2593-2600. https://doi.org/10. 1093/humrep/del221

33. Balasch J, Fábregues F (2006) LH in the follicular phase: neither too high nor too low. Reprod BioMed Online 12(4):406-415. https://doi.org/10. 1016/s1472-6483(10)61991-8

34. White DM, Polson DW, Kiddy D, Sagle P, Watson H, Gilling-Smith C et al (1996) Induction of ovulation with low-dose gonadotropins in polycystic ovary syndrome: an analysis of 109 pregnancies in 225 women. J Clin Endocrinol Metab 81(11):3821-3824. https://doi.org/10.1210/jcem.81.11. 8923819

35. J B. (2004) The role of FSH and LH in ovulation induction: current concepts and the contribution of recombinant gonadotropins. In: Gardner DK, Weissman A, Howles CM, Shoham Z (eds) Textbook of Assisted Reproductive Techniques: Laboratory and Clinical Perspectives 2nd edn. Taylor and Francis, London, pp 541-565

36. Simoni MNE (1995) FSH in therapy: physiological basis, new preparations and clinical use. Reprod Med Rev 4:163-177

37. Taymor ML (1996) The regulation of follicle growth: some clinical implications in reproductive endocrinology. Fertil Steril 65(2):235-247. https:// doi.org/10.1016/s0015-0282(16)58077-7

38. Hugues JN, Soussis J, Calderon I, Balasch J, Anderson RA, Romeu A (2005) Does the addition of recombinant $\mathrm{LH}$ in WHO group II anovulatory women over-responding to FSH treatment reduce the number of developing follicles? A dose-finding study. Hum Reprod 20(3):629-635. https:// doi.org/10.1093/humrep/deh682

39. Loumaye E, Engrand P, Shoham Z, Hillier SG, Baird DT (2003) Clinical evidence for an LH 'ceiling' effect induced by administration of recombinant human LH during the late follicular phase of stimulated cycles in World Health Organization type I and type II anovulation. Hum Reprod 18(2):314-322. https://doi.org/10.1093/humrep/deg066

40. Alviggi C, Conforti A, De Rosa P, Strina I, Palomba S, Vallone R et al (2017) The distribution of stroma and antral follicles differs between insulinresistance and hyperandrogenism-related polycystic ovarian syndrome. Front Endocrinol (Lausanne) 8:117. https://doi.org/10.3389/fendo.2017. 00117

41. Copperman AB, Benadiva C (2013) Optimal usage of the GnRH antagonists: a review of the literature. Reprod Biol Endocrinol 11:20. https://doi org/10.1186/1477-7827-11-20

42. Xiong Y, Bu Z, Dai W, Zhang M, Bao X, Sun Y (2014) Recombinant luteinizing hormone supplementation in women undergoing in vitro fertilization/intracytoplasmic sperm injection with gonadotropin releasing hormone antagonist protocol: a systematic review and metaanalysis. Reprod Biol Endocrinol 12(1):109. https://doi.org/10.1186/ 1477-7827-12-109

43. Baruffi RL, Mauri AL, Petersen CG, Felipe V, Martins AM, Cornicelli J et al (2007) Recombinant LH supplementation to recombinant FSH during induced ovarian stimulation in the $\mathrm{GnRH}$-antagonist protocol: a metaanalysis. Reprod BioMed Online 14(1):14-25. https://doi.org/10.1016/ s1472-6483(10)60758-4

44. Kolibianakis EM, Kalogeropoulou L, Griesinger G, Papanikolaou EG, Papadimas J, Bontis J et al (2007) Among patients treated with FSH and $\mathrm{GnRH}$ analogues for in vitro fertilization, is the addition of recombinant 
LH associated with the probability of live birth? A systematic review and meta-analysis. Hum Reprod Update 13(5):445-452. https://doi.org/10. 1093/humupd/dmm008

45. Mochtar MH, Danhof NA, Ayeleke RO, Van der Veen F, van Wely M (2017) Recombinant luteinizing hormone (rLH) and recombinant follicle stimulating hormone (rFSH) for ovarian stimulation in IVF/ICSI cycles. Cochrane Database Syst Rev 5(5):Cd005070. https://doi.org/10.1002/14651858. CD005070.pub3

46. Oliveira JB, Mauri AL, Petersen CG, Martins AM, Cornicelli J, Cavanha M et al (2007) Recombinant luteinizing hormone supplementation to recombinant follicle-stimulation hormone during induced ovarian stimulation in the GnRH-agonist protocol: a meta-analysis. J Assist Reprod Genet 24(2-3):67-75. https://doi.org/10.1007/s10815-006-9095-4

47. Santi D, Casarini L, Alviggi C, Simoni M (2017) Efficacy of follicle-stimulating hormone (FSH) alone, FSH + luteinizing hormone, human menopausal gonadotropin or FSH + human chorionic gonadotropin on assisted reproductive technology outcomes in the "personalized" medicine era: a meta-analysis. Front Endocrinol (Lausanne) 8:114. https://doi.org/10. 3389/fendo.2017.00114

48. Hill MJ, Levens ED, Levy G, Ryan ME, Csokmay JM, DeCherney AH et al (2012) The use of recombinant luteinizing hormone in patients undergoing assisted reproductive techniques with advanced reproductive age: a systematic review and meta-analysis. Fertil Steril 97(5):1108-1114.e1. https://doi.org/10.1016/j.fertnstert.2012.01.130

49. Lehert $P$, Kolibianakis EM, Venetis CA, Schertz J, Saunders H, Arriagada P et al (2014) Recombinant human follicle-stimulating hormone (r-hFSH) plus recombinant luteinizing hormone versus $\mathrm{r}$-hFSH alone for ovarian stimulation during assisted reproductive technology: systematic review and meta-analysis. Reprod Biol Endocrinol 12:17. https://doi.org/10.1186/ 1477-7827-12-17

50. Acevedo B, Sanchez M, Gomez JL, Cuadros J, Ricciarelli E، Hernández ER (2004) Luteinizing hormone supplementation increases pregnancy rates in gonadotropin-releasing hormone antagonist donor cycles. Fertil Steril 82(2):343-347. https://doi.org/10.1016/j.fertnstert.2004.03.020

51. König TE, van der Houwen LE, Overbeek A, Hendriks ML, Beutler-Beemsterboer SN, Kuchenbecker WK et al (2013) Recombinant LH supplementation to a standard GnRH antagonist protocol in women of 35 years or older undergoing IVF/ICSI: a randomized controlled multicentre study. Hum Reprod 28(10):2804-2812. https://doi.org/10.1093/humrep/det266

52. Bosch E, Labarta E, Crespo J, Simón C, Remohí J, Pellicer A (2011) Impact of luteinizing hormone administration on gonadotropin-releasing hormone antagonist cycles: an age-adjusted analysis. Fertil Steril 95(3):10311036. https://doi.org/10.1016/j.fertnstert.2010.10.021

53. Chen LCC-R, Kong X-J, Qiu C-R (2019) Effects of luteinizing hormone supplementation on ovarian response and assisted reproductive technology outcomes in antagonist in vitro fertilization/intracytoplasmic sperm injection cycles: a meta-analysis. Reprod Dev Med 3(2):88-98

54. Levi-Setti PE, Cavagna M, Bulletti C (2006) Recombinant gonadotrophins associated with $\mathrm{GnRH}$ antagonist (cetrorelix) in ovarian stimulation for ICSI: comparison of $\mathrm{r}-\mathrm{FSH}$ alone and in combination with r-LH. Eur J Obstet Gynecol Reprod Biol 126(2):212-216. https://doi.org/10.1016/j. ejogrb.2005.11.023

55. Humaidan P (2006) To add or not to add LH: comments on a recent commentary. Reprod BioMed Online 12(3):284-285. https://doi.org/10.1016/ s1472-6483(10)60998-4

56. SK (2005) To add or not to add LH: consideration of LH concentration changes in individual patients 11(6):664-6

57. Vuong TN, Phung HT, Ho MT (2015) Recombinant follicle-stimulating hormone and recombinant luteinizing hormone versus recombinant folliclestimulating hormone alone during $\mathrm{GnRH}$ antagonist ovarian stimulation in patients aged $\geq 35$ years: a randomized controlled trial. Hum Reprod 30(5):1188-1195. https://doi.org/10.1093/humrep/dev038

58. Younis JS, Izhaki I, Ben-Ami M (2016) The effect of LH supplementation to the $\mathrm{GnRH}$ antagonist protocol in advanced reproductive ageing women: a prospective randomized controlled study. Clin Endocrinol 84(1):99-106. https://doi.org/10.1111/cen.12886

59. Gómez-Palomares JL, Acevedo-Martín B, Andrés L, Ricciarelli E, Hernández ER (2005) LH improves early follicular recruitment in women over 38 years old. Reprod BioMed Online 11(4):409-414. https://doi.org/10.1016/s14726483(10)61131-5
60. Conforti A, Esteves SC, Humaidan P, Longobardi S, D'Hooghe T, Orvieto $R$ et al (2021) Recombinant human luteinizing hormone co-treatment in ovarian stimulation for assisted reproductive technology in women of advanced reproductive age: a systematic review and meta-analysis of randomized controlled trials. Reprod Biol Endocrinol 19(1):91. https://doi. org/10.1186/s12958-021-00759-4

61. Ruvolo G, Bosco L, Pane A, Morici G, Cittadini E, Roccheri MC (2007) Lower apoptosis rate in human cumulus cells after administration of recombinant luteinizing hormone to women undergoing ovarian stimulation for in vitro fertilization procedures. Fertil Steril 87(3):542-546. https://doi.org/ 10.1016/j.fertnstert.2006.06.059

62. Ferraretti AP, Gianaroli L, Magli MC, D'Angelo A, Farfalli V, Montanaro N (2004) Exogenous luteinizing hormone in controlled ovarian hyperstimulation for assisted reproduction techniques. Fertil Steril 82(6):1521-1526. https://doi.org/10.1016/j.fertnstert.2004.06.041

63. De Placido G, Alviggi C, Perino A, Strina I, Lisi F, Fasolino A et al (2005) Recombinant human $\mathrm{LH}$ supplementation versus recombinant human FSH (rFSH) step-up protocol during controlled ovarian stimulation in normogonadotrophic women with initial inadequate ovarian response to $\mathrm{rFSH}$. A multicentre, prospective, randomized controlled trial. Hum Reprod 20(2):390-396. https://doi.org/10.1093/humrep/deh625

64. Conforti A, Esteves SC, Di Rella F, Strina I, De Rosa P, Fiorenza A et al (2019) The role of recombinant $\mathrm{LH}$ in women with hypo-response to controlled ovarian stimulation: a systematic review and meta-analysis. Reprod Biol Endocrinol 17(1):18. https://doi.org/10.1186/s12958-019-0460-4

65. Sheikhha MH, Eftekhar M, Kalantar SM (2011) Investigating the association between polymorphism of follicle-stimulating hormone receptor gene and ovarian response in controlled ovarian hyperstimulation. J Hum Reprod Sci 4(2):86-90. https://doi.org/10.4103/0974-1208.86089

66. Loutradis D, Patsoula E, Minas V, Koussidis GA, Antsaklis A, Michalas S et al (2006) FSH receptor gene polymorphisms have a role for different ovarian response to stimulation in patients entering IVF/ICSI-ET programs. J Assist Reprod Genet 23(4):177-184. https://doi.org/10.1007/s10815-005-9015-z

67. Ferraretti AP, La Marca A, Fauser BC, Tarlatzis B, Nargund G, Gianaroli L (2011) ESHRE consensus on the definition of 'poor response' to ovarian stimulation for in vitro fertilization: the Bologna criteria. Hum Reprod 26(7):1616-1624. https://doi.org/10.1093/humrep/der092

68. Berkkanoglu M, Isikoglu M, Aydin D, Ozgur K (2007) Clinical effects of ovulation induction with recombinant follicle-stimulating hormone supplemented with recombinant luteinizing hormone or low-dose recombinant human chorionic gonadotropin in the midfollicular phase in microdose cycles in poor responders. Fertil Steril 88(3):665-669. https://doi.org/10.1016/j.fertnstert.2006.11.150

69. Gizzo S, Andrisani A, Noventa M, Manfè S, Oliva A, Gangemi M et al (2015) Recombinant LH supplementation during IVF cycles with a GnRH-antagonist in estimated poor responders: a cross-matched pilot investigation of the optimal daily dose and timing. Mol Med Rep 12(3):4219-4229. https://doi.org/10.3892/mmr.2015.3904

70. Raju GA, Chavan R, Deenadayal M, Gunasheela D, Gutgutia R, Haripriya $G$ et al (2013) Luteinizing hormone and follicle stimulating hormone synergy: a review of role in controlled ovarian hyper-stimulation. J Hum Reprod Sci 6(4):227-234. https://doi.org/10.4103/0974-1208.126285

71. Musters AM, van Wely M, Mastenbroek S, Kaaijk EM, Repping S, van der Veen F et al (2012) The effect of recombinant LH on embryo quality: a randomized controlled trial in women with poor ovarian reserve. Hum Reprod 27(1):244-250. https://doi.org/10.1093/humrep/der371

72. Barrenetxea G, Agirregoikoa JA, Jiménez MR, de Larruzea AL, Ganzabal T, Carbonero K (2008) Ovarian response and pregnancy outcome in poor-responder women: a randomized controlled trial on the effect of luteinizing hormone supplementation on in vitro fertilization cycles. Fertil Steril 89(3):546-553. https://doi.org/10.1016/j.fertnstert.2007.03.088

73. Ferraretti AP, Gianaroli L, Motrenko T, Feliciani E, Tabanelli C, Magli MC (2014) LH pretreatment as a novel strategy for poor responders. BioMed research international. 2014:926172. https://doi.org/10.1155/2014/ 926172

74. Humaidan P, Alviggi C, Fischer R, Esteves SC (2016) The novel POSEIDON stratification of 'Low prognosis patients in Assisted Reproductive Technology'and its proposed marker of successful outcome. F1000Res 5:2911. https://doi.org/10.12688/f1000research.10382.1

75. Conforti A, Esteves SC, Picarelli S, lorio G, Rania E, Zullo F et al (2019) Novel approaches for diagnosis and management of low prognosis patients in 
assisted reproductive technology: the POSEIDON concept. Panminerva Med 61(1):24-29. https://doi.org/10.23736/s0031-0808.18.03511-5

76. Zeleznik AJF-BD (1994) Control of follicular development, corpus luteum funcion, and the recognition of pregnancy in higher primates. The physiology of reproduction. 2nd ed, Vol 2. Raven Press, New York, pp 751-782

77. Filicori M, Cognigni GE, Pocognoli P, Tabarelli C, Spettoli D, Taraborrelli S et al (2002) Modulation of folliculogenesis and steroidogenesis in women by graded menotrophin administration. Hum Reprod 17(8):2009-2015. https://doi.org/10.1093/humrep/17.8.2009

78. (2001) Human recombinant luteinizing hormone is as effective as, but safer than, urinary human chorionic gonadotropin in inducing final follicular maturation and ovulation in in vitro fertilization procedures: results of a multicenter double-blind study. J Clin Endocrinol Metab 86(6):26072618. https://doi.org/10.1210/jcem.86.6.7599

79. Caserta D, Lisi F, Marci R, Ciardo F, Fazi A, Lisi R et al (2011) Does supplementation with recombinant luteinizing hormone prevent ovarian hyperstimulation syndrome in down regulated patients undergoing recombinant follicle stimulating hormone multiple follicular stimulation for IVF/ET and reduces cancellation rate for high risk of hyperstimulation? Gynecol Endocrinol 27(11):862-866. https://doi.org/10.3109/09513590. 2010.544133

80. Orvieto $\mathrm{R}$ (2019) HMG versus recombinant FSH plus recombinant $\mathrm{LH}$ in ovarian stimulation for IVF: does the source of LH preparation matter? Reprod BioMed Online 39(6):1001-1006. https://doi.org/10.1016/j.rbmo. 2019.08.010

81. Trinchard-Lugan I, Khan A, Porchet HC, Munafo A (2002) Pharmacokinetics and pharmacodynamics of recombinant human chorionic gonadotrophin in healthy male and female volunteers. Reprod BioMed Online 4(2):106-115. https://doi.org/10.1016/s1472-6483(10)61927-x

82. Barberi M, Ermini B, Morelli MB, Ermini M, Cecconi S, Canipari R (2012) Follicular fluid hormonal profile and cumulus cell gene expression in controlled ovarian hyperstimulation with recombinant FSH: effects of recombinant LH administration. J Assist Reprod Genet 29(12):1381-1391. https://doi.org/10.1007/s10815-012-9893-9

83. Gatta V, Tatone C, Ciriminna R, Vento M, Franchi S, d'Aurora M et al (2013) Gene expression profiles of cumulus cells obtained from women treated with recombinant human luteinizing hormone + recombinant human follicle-stimulating hormone or highly purified human menopausal gonadotropin versus recombinant human follicle-stimulating hormone alone. Fertil Steril 99(7):2000-2008.e1. https://doi.org/10.1016/j.fertnstert. 2013.01.150

84. Mak SM, Wong WY, Chung HS, Chung PW, Kong GW, Li TC et al (2017) Effect of mid-follicular phase recombinant LH versus urinary HCG supplementation in poor ovarian responders undergoing IVF - a prospective double-blinded randomized study. Reprod BioMed Online 34(3):258-266. https://doi.org/10.1016/j.rbmo.2016.11.014

85. Trew GH, Brown AP, Gillard S, Blackmore S, Clewlow C, O'Donohoe P et al (2010) In vitro fertilisation with recombinant follicle stimulating hormone requires less IU usage compared with highly purified human menopausal gonadotrophin: results from a European retrospective observational chart review. Reprod Biol Endocrinol 8:137. https://doi.org/10.1186/ 1477-7827-8-137
86. van Wely M, Kwan I, Burt AL, Thomas J, Vail A, Van der Veen F et al (2012) Recombinant versus urinary gonadotrophin for ovarian stimulation in assisted reproductive technology cycles. A Cochrane review. Hum Reprod Update 18(2):111. https://doi.org/10.1093/humupd/dmr048

87. Camp TA, Rahal JO, Mayo KE (1991) Cellular localization and hormona regulation of follicle-stimulating hormone and luteinizing hormone receptor messenger RNAs in the rat ovary. Mol Endocrinol (Baltimore, Md) 5(10):1405-1417. https://doi.org/10.1210/mend-5-10-1405

88. Yung Y, Aviel-Ronen S, Maman E, Rubinstein N, Avivi C, Orvieto R et al (2014) Localization of luteinizing hormone receptor protein in the human ovary. Mol Hum Reprod 20(9):844-849. https://doi.org/10.1093/molehr/ gau041

89. Alviggi C, Pettersson K, Longobardi S, Andersen CY, Conforti A, De Rosa P et al (2013) A common polymorphic allele of the LH beta-subunit gene is associated with higher exogenous FSH consumption during controlled ovarian stimulation for assisted reproductive technology. Reprod Biol Endocrinol 11:51. https://doi.org/10.1186/1477-7827-11-51

90. Papamentzelopoulou M, Mavrogianni D, Partsinevelos GA, Marinopoulos S, Dinopoulou V, Theofanakis C et al (2012) LH receptor gene expression in cumulus cells in women entering an ART program. J Assist Reprod Genet 29(5):409-416. https://doi.org/10.1007/s10815-012-9729-7

91. Yin Q, Li Y, Huang J, Yang D (2015) Association of rs 13405728 polymorphism of LHR gene with slow ovarian response. Zhonghua Yi Xue Yi Chuan Xue Za Zhi 32(6):840-843. https://doi.org/10.3760/cma.j.issn.10039406.2015.06.020

92. Maman E, Yung Y, Kedem A, Yerushalmi GM, Konopnicki S, Cohen B et al (2012) High expression of luteinizing hormone receptors messenger RNA by human cumulus granulosa cells is in correlation with decreased fertilization. Fertil Steril 97(3):592-598. https://doi.org/10.1016/j.fertnstert. 2011.12.027

93. Desai SS, Achrekar SK, Paranjape SR, Desai SK, Mangoli VS, Mahale SD (2013) Association of allelic combinations of FSHR gene polymorphisms with ovarian response. Reprod BioMed Online 27(4):400-406. https://doi. org/10.1016/j.rbmo.2013.07.007

94. Lindgren I, Bååth M, Uvebrant K, Dejmek A, Kjaer L, Henic E et al (2016) Combined assessment of polymorphisms in the LHCGR and FSHR genes predict chance of pregnancy after in vitro fertilization. Hum Reprod 31(3):672-683. https://doi.org/10.1093/humrep/dev342

95. Lu X, Yan Z, Cai R, Khor S, Wu L, Sun L et al (2019) Pregnancy and live birth in women with pathogenic LHCGR variants using their own oocytes. J Clin Endocrinol Metab 104(12):5877-5892. https://doi.org/10.1210/jc. 2019-01276

96. Alviggi C, Conforti A, Santi D, Esteves SC, Andersen CY, Humaidan P et al (2018) Clinical relevance of genetic variants of gonadotrophins and their receptors in controlled ovarian stimulation: a systematic review and meta-analysis. Hum Reprod Update 24(5):599-614. https://doi.org/10. 1093/humupd/dmy019

\section{Publisher's Note}

Springer Nature remains neutral with regard to jurisdictional claims in published maps and institutional affiliations.

\section{Submit your manuscript to a SpringerOpen ${ }^{\circ}$ journal and benefit from:}

- Convenient online submission

- Rigorous peer review

- Open access: articles freely available online

- High visibility within the field

- Retaining the copyright to your article

Submit your next manuscript at $\boldsymbol{\nabla}$ springeropen.com 